\title{
Intervención grupal para reducir la violencia de género entre consumidoras de drogas. Resultados de un estudio piloto en un centro comunitario de tratamiento de adicciones
}

\author{
A group intervention to reduce intimate partner violence \\ among female drug users. Results from a randomized \\ controlled pilot trial in a community substance-abuse center
}

\author{
Judit Tirado-Muñoz*, Gail Gilchrist*,***, Eva Lligoña**, Louisa Gilbert****, Marta Torrens *,** \\ *Addiction Research Group, IMIM-Institut Hospital del Mar d'Investigacions Mèdiques; Universitat Autónoma de Barcelona, \\ Barcelona, Spain \\ ** Institute of Neuropsychiatry and Addictions, Parc de Salut Mar, Barcelona, Spain \\ *** National Addiction Centre, Institute of Psychiatry, King's College, London, UK \\ **** Social Intervention Group, Columbia University, New York, NY, USA
}

\section{Resumen}

Antecedentes: Las mujeres usuarias de drogas son víctimas de violencia de género en mayor proporción que las mujeres en población general; sin embargo, pocas intervenciones se han desarrollado para reducir la violencia de género entre mujeres usuarias de drogas. Métodos: Una versión adaptada de la intervención "Women's Wellness Treatment" para reducir violencia de género y síntomas depresivos, ha sido evaluada mediante un ensayo clínico piloto en un centro de tratamiento ambulatorio en Barcelona, España. Catorce mujeres que recibían tratamiento ambulatorio para un trastorno por consumo de sustancias y que declararon ser víctimas de violencia en el mes anterior fueron incluidas en el ensayo clínico. Las participantes fueron asignados al azar para recibir 10 sesiones grupales de terapia cognitivo-conductual (IPaViT-CBT) o tratamiento habitual. La frecuencia de violencia, síntomas depresivos, consumo de sustancias, calidad de vida y estado de salud fueron evaluados al inicio del estudio y 1 , 3 y 12 meses después de la intervención. Se realizó análisis por intención de tratar. Resultados: Se encontraron efectos moderados de la intervención en la reducción de maltrato psicológico, aumento de la asertividad y la reducción de la agresividad en la relación de pareja, y reducción en la frecuencia de consumo de alcohol hasta 3 meses después de la intervención. La intervención no redujo significativamente la probabilidad de ser víctima de cualquier tipo de violencia de género, los síntomas depresivos, calidad de vida o el estado de salud auto-referido, hasta 12 meses después de la intervención. Conclusiones: Los resultados de este estudio piloto indican que es factible realizar una intervención de 10 sesiones grupales de TCC entre las víctimas de violencia de género que reciben tratamiento por uso de sustancias en un centro comunitario de tratamiento de adicciones. Se requiere un ensayo clínico más robusto para replicar estos resultados. Palabras clave: violencia de género; abuso de substancias, mujeres, terapia cognitivo-conductual, intervención grupal, ensayo aleatorizado controlado.

\begin{abstract}
Background: A greater proportion of drug dependent women are victims of intimate partner violence (IPV) than women in the general population; however, few interventions have been developed to reduce IPV among drug dependent women. Methods: An adapted version of the Women's Wellness Treatment, to address IPV and depressive symptoms, was piloted in a randomized controlled trial conducted in outpatient treatment program in Barcelona, Spain among 14 women receiving outpatient treatment for a drug use disorder who screened positive for IPV in the previous month. Participants were randomly assigned to receive the 10 session cognitive behavioral therapy (IPaViTCBT) group intervention or treatment as usual. The frequency of IPV, depressive symptoms, substance use, quality of life and health status were assessed at baseline and 1, 3 and 12 months post intervention. Intention to treat analysis was performed. Results: Moderate effects for the intervention were found in reducing psychological maltreatment, increasing assertiveness of IPV and reducing aggressiveness in the partner relationship, and in reducing the frequency of drinking up to 3 months post intervention. The intervention did not significantly reduce the likelihood of any IPV, depressive symptoms, quality of life or self-reported health status, up to 12-months post intervention. Conclusion: This pilot trial suggests some initial support for the 10-session CBT group intervention among IPV victims who received treatment for drug use. Study findings indicate that it is feasible to deliver the intervention in a community substance abuse center. An adequately powered trial is required to replicate these results. Keywords: intimate partner violence; substance abuse; females; cognitive behavioral therapy; group intervention; randomized controlled trial.
\end{abstract}

Recibido: Julio 2014; Aceptado: Diciembre 2014

Enviar correspondecia a:

Marta Torrens MD, PhD. Addiction Unit. Institute of Neuropsychiatry and Addictions, Parc de Salut Mar, Passeig Marítim, 25-29, 08003 Barcelona, Spain. E-mail: mtorrens@parcdesalutmar.cat 


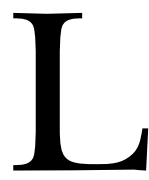
a violencia de género (VG) produce creciente preocupación en el ámbito de la salud pública. La Organización Mundial de la Salud (OMS) estima que un tercio de las mujeres a nivel mundial han sido víctimas de la VG por lo menos una vez en su vida (OMS, 2013). Las tasas de victimización por VG física y sexual son aún mayores entre las mujeres con un trastorno por uso de sustancias (TUS) (Cohen y Hien, 2006; El-Bassel, Gilbert, Witte, Wu y Chang, 2011; El-Bassel, Gilbert, Wu, Go y Hill, 2005; Miller, Downs y Testa, 1993), oscilando entre un 40-70\% entre las mujeres en tratamiento por abuso de sustancias (Gilbert et al., 2006; Gilchrist, Blazquez y Torrens, 2011; Wagner et al., 2009). La victimización por VG está fuertemente asociada a problemas de salud mental (Campbell, 2002; Howard et al., 2010; Trevillion, Oram, Feder y Howard, 2012), y la evidencia reciente confirma que alrededor del $20 \%$ de las mujeres que experimentaron VG en el último año desarrollaron un nuevo trastorno psiquiátrico (Okuda et al., 2011). La alta tasa de VG entre las usuarias de sustancias tiene consecuencias adversas sobre su salud física, mental y reproductiva. Entre las usuarias de drogas, la VG está asociada a problemas de salud mental (Gilchrist, Blazquez y Torrens, 2012), incluyendo la depresión. Las víctimas de VG con TUS son más propensas a informar de síntomas depresivos ( $\mathrm{OR}=2.35)$ (Connelly, Hazen, Baker-Ericzen, Landsverk y Horwitz, 2013; Illangasekare, Burke, McDonnell y Gielen, 2013) y a cumplir los criterios para el Trastorno de estrés postraumático (TEPT) $(\mathrm{OR}=2.7)$ (Cohen, Field, Campbell y Hien, 2013; Kaysen et al., 2007; Najavits, Sonn, Walsh y Weiss, 2004; Peters, Khondkaryan y Sullivan, 2012) que las mujeres con TUS que no hayan experimentado VG. Un estudio reciente de población general en Chile encontró que la prevalencia de ser víctima de asalto, agresión o violencia sexual era mayor entre los hombres y las mujeres con un mayor consumo mensual de alcohol o quienes consumían alcohol excesivamente (seis o más copas de una vez al menos una vez al mes) comparados con aquellos que no presentaban estos patrones de consumo (Castillo-Carniglia, Pizarro, Luengo y Soto-Brandt, 2014). Además, la VG conlleva aumentar o mantener el abuso de sustancias (El-Bassel, Gilbert, Wu, Go y Hill, 2005; McKinney, Caetano, Rodriguez y Okoro, 2010; Testa, Livingston y Hoffman, 2007) y a más enfermedades físicas y mayor utilización de recursos sanitarios (Liebschutz, Mulvey y Samet, 1997). Las mujeres con TUS que sufren VG pueden participar en relaciones sexuales y prácticas de inyección no seguras, potencialmente debido a la influencia negativa del agresor (Wagner et al., 2009) lo cual puede exponerlas a mayor riesgo de virus transmitidos por sangre, infecciones sexuales y embarazos no deseados (Bourgois, Prince y Moss, 2004; Campbell et al., 2008; El-Bassel, Gilbert, Witte, Wu y Chang, 2011; Singer, 1996). Como tal, las intervenciones deben considerar las relaciones de las mujeres con TUS con sus parejas (Hearn, O'Sullivan, El-Bassel y Gilbert, 2005) y abordar todos los aspectos de sus vidas, incluyendo los TUS, la comorbilidad psiquiátrica y los comportamientos de riesgo sexuales y de inyección. Revisiones sistemáticas previas han identificado las intervenciones que abordan simultáneamente trauma y abuso de sustancias (Najavits, 2009) y el TUS entre mujeres supervivientes de VG (Fowler y Faulkner, 2011). Una revisión sistemática más reciente sobre intervenciones evaluadas mediante ensayos clínicos aleatorizados (EAC) para reducir la VG entre las mujeres (Tirado-Muñoz, Gilchrist, Farré, Hegarty y Torrens, 2014), sólo identificó una intervención dirigida a mujeres con TUS donde VG fue un resultado evaluado: el Women's Wellness Treatment- WWT (en español: El Tratamiento del Bienestar de las Mujeres; Gilbert et al., 2006). Se identificaron otras intervenciones que abordan el uso de sustancias, los síntomas de PTSD y la VG tales como ATRIUM (Addiction and Trauma Recovery Integration Model- en español: Modelo de Integración de Adicción y Recuperación de Trauma; Miller y Guidry, 2001), TREM (Trauma Recovery and Empowerment Model-en español: Modelo de Recuperación de Trauma y Empoderamiento; Harris, 1998), Seeking Safety (SS: A treatment manual for PTSD and Substance Abuse; en español: Búsqueda de Seguridad: un manual de tratamiento para el estrés postraumático y abuso de sustancias; Najavits, 2002) y TRIAD, más centrado en la enseñanza de habilidades interpersonales (Fearday, Clark y Edington, 2001). La Búsqueda de Seguridad (Seeking Safety) es claramente la intervención para PTSD-TUS más estudiada. Por último, la WWT fue la intervención más centrada en el propósito de esta investigación en términos del resultado primario y la intensidad de la intervención.

El propósito de este ensayo piloto fue: (1) adaptar la intervención de grupo manualizada WWT para abordar la VG y los síntomas depresivos entre las mujeres que reciben tratamiento para uso de sustancias ; (2) llevar a cabo a un ensayo clínico piloto para evaluar la viabilidad y la eficacia inicial de la intervención de terapia cognitivo-conductual (TCC) en la reducción de la VG en comparación con el tratamiento habitual 1, 3 y 12 meses después de la intervención.

\section{Métodos}

\section{Descripción del diseño y de la muestra de estudio}

El ensayo clínico se llevó a cabo desde marzo de 2011 a junio de 2012 en un centro comunitario de tratamiento ambulatorio de adicciones en Barcelona (España). La aprobación ética fue otorgada por el Comité de Ética en la Investigación Humana del Instituto IMIM-Institut Hospital del Mar d'Investigacions Mèdiques; Parc de Salut Mar de Barcelona.

Las participantes se reclutaron de las salas de espera de dos centros de tratamiento ambulatorio de drogadicción en Barcelona. La investigadora abordó a las participantes potenciales en la sala de espera, les explicó los objetivos del estudio y les entregó un folleto informativo. Si accedían a participar, fueron sometidas a un cribado para determinar si cumplían los criterios de inclusión del estudio. Las mujeres con TUS eran elegi- 


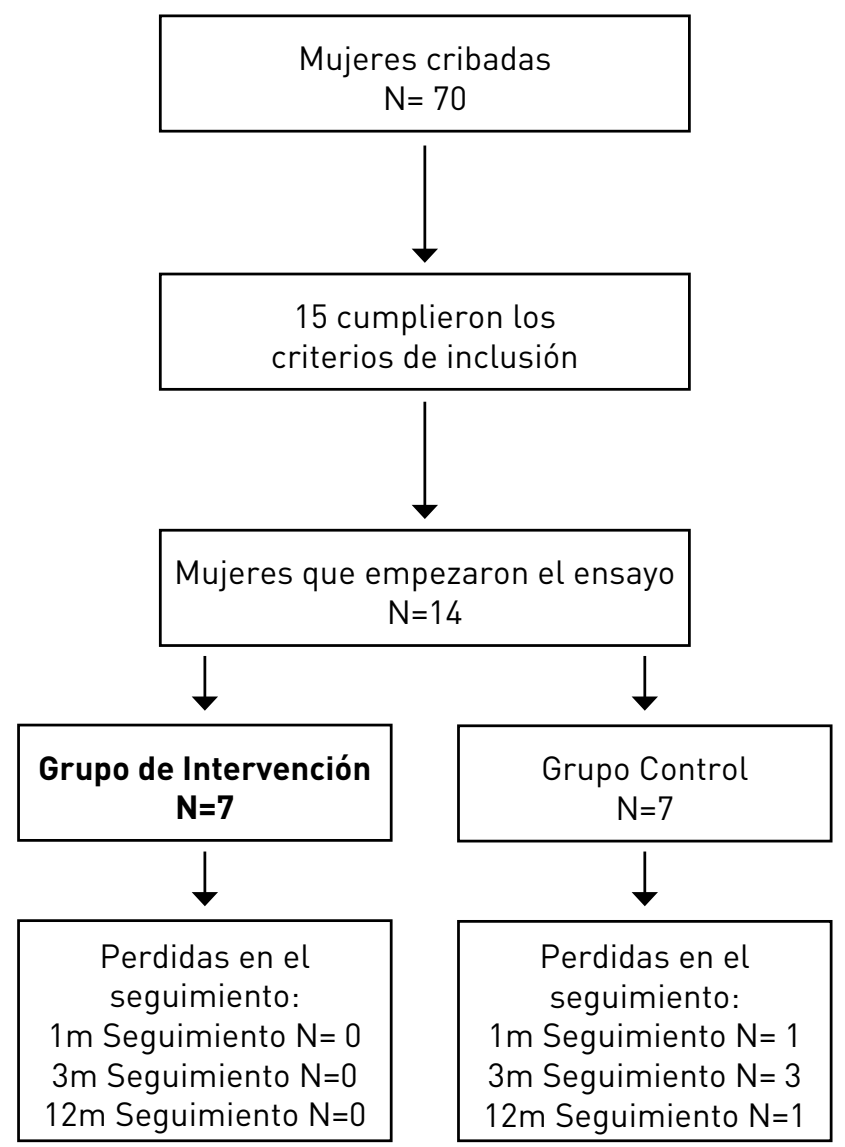

Figura 1. Diagrama de flujo del estudio

bles para el estudio si: a) tenían 18 años o más; b) estaban recibiendo tratamiento para el abuso de sustancias en un centro de tratamiento ambulatorio de adicciones; c) se encontraban actualmente en una relación con una pareja masculina; d) habían informado de VG en el último mes, utilizando una versión adaptada de la Composite Abuse Scale (Escala Compuesta de Abuso; Hegarty, Sheehan y Sconfeld, 1999) y la Psychological Maltreatment of Women Inventory (Inventario de Maltrato Psicológico de Mujeres; Tolman, 1999); y e) podían comunicarse en español. Setenta mujeres con TUS fueron evaluadas, de las que 15 eran elegibles para participar, y 14 de ellas aceptaron participar. Ninguna de las participantes recibió un incentivo financiero por completar las evaluaciones de seguimiento. De las 14 participantes que comenzaron el ensayo después de la aleatorización, se perdieron tres para el seguimiento - una se mudó fuera de Barcelona, una recibió tratamiento hospitalario, y una fue encarcelada (Figura 1).

\section{Intervención: Proceso de adaptación}

La revisión sistemática de las intervenciones evaluadas mediante EAC para reducir la VG entre las mujeres (Tirado-Muñoz, Gilchrist, Farré, Hegarty y Torrens, 2014) sólo identificó una intervención dirigida a usuarias de sustancias, la WWT (Gilbert et al., 2006). Con el consentimiento de los autores, la intervención WWT fue traducida al español y adaptada a las necesidades de mujeres con TUS y al sistema de tratamiento de adicciones en España. La intervención original WWT ofrecía 11 sesiones grupales de 2 horas, más 1 sesión individual, dirigida a promover la seguridad en la relación y a reducir el consumo desustancias. Basado en la experiencia previa en España de ofrecer intervenciones de grupo y en la retención de las participantes, decidimos reducir el número de sesiones de 12 a 10 y abordar el estado de ánimo negativo, dada la alta prevalencia de depresión entre las mujeres en tratamiento de adicciones (Torrens, Gilchrist y Domingo-Salvany, 2011). Por lo tanto, se incluyó una nueva sesión abordando el estado de ánimo negativo, que fue adaptado con el permiso de los autores de la Behavioural Therapy for Depression in Drug Dependence Manual (BTDD; en español, Manual de terapia conductual para la depresión en dependencia de drogas; Carpenter, Aharonovich, Smith, Iguchi y Nunes, 2006). En esta sesión, se presentó y se comentó el modelo de la depresión, y se puso de relieve la importancia de la satisfacción con la vida, alentando a las mujeres a introducir actividades agradables en su vida y ayudándoles a desarrollar una lista de dichas actividades. A las mujeres del ensayo se les ofreció la oportunidad de participar (sin coste) en las clases de su elección en un centro comunitario local (p. e., yoga, danza, cocina, informática, etc.) en un intento de facilitar actividades agradables y a su vez reducir el estado de ánimo negativo. Debido a la alta prevalencia de hepatitis C entre las mujeres que se inyectan drogas (Gilchrist, Blazquez y Torrens, 2011) adaptamos la sesión original sobre el VIH para incluir educación sobre la transmisión de la hepatitis C.

El equipo de investigación en España realizó la fase de adaptación, en consulta con los autores originales de ambos tratamientos manualizados (Carpenter, Aharonovich, Smith, Iguchi y Nunes, 2006; Gilbert et al., 2006). La intervención final IPaViT-CBT (Terapia dirigida a la Violencia de Género- Terapia Cognitivo Conductual) consistió en 10 sesiones semanales de grupo de 2 horas de duración. Las siguientes 10 sesiones se administraron en formato de grupo:

- Sesión 1 Preparación para el viaje: mejorar la motivación por el bienestar

- Sesión 2 Construir una relación de seguridad (sesión de grupo)

- Sesión 3 Identificar los desencadenantes del consumo de drogas y de conflictos en la relación

- Sesión 4 Curarse de la VG psicológica

- Sesión 5 Manejar la VG física: la reconstrucción de la ira

- Sesión 6 Recuperación del trauma: identificar los desencadenantes del TEPT

- Sesión 7 Manejar el estado de ánimo negativo

- Sesión 8 Establecer límites sexuales: habilidades de negociación

- Sesión 9 Evitar las relaciones sexuales peligrosas: identificación de factores desencadenantes para el riesgo de VIH / VHC e identificar estrategias para reducir el riesgo de VIH / VHC.

- Sesión 10 En el camino a la recuperación y la seguridad: celebrando éxitos. 
El formato de la intervención a lo largo de las sesiones consistió en una secuencia de 5 pasos. Cada sesión se inicia con: (1) una apertura inspiradora (una cita de una artista o una escritora) que sirve para inspirar y motivar a las participantes hacia su recuperación y formando parte del contexto de la sesión; (2) se completó un registro de recuperación y de relación para cada participante para posteriormente detectar y comentar cualquier incidente que ocurra entre las sesiones relacionado con el consumo de sustancias o con conflictos de pareja, identificando los desencadenantes para ayudar a las mujeres a generar planes de seguridad y ofrecerles nuevas habilidades para reducir la VG; (3) sensibilizar, a través de la discusión sobre la relación entre los comportamientos asociados con las drogas y diversas formas de VG; (4) construir habilidades y discusión grupal; y (5) un chequeo de las necesidades / problemas más destacados de las participantes y cómo hacer frente a éstos. Al final de cada sesión, se animó a las participantes a hacer 'tareas para casa' entre sesiones (p. e., autoafirmaciones positivas, activación conductual). La intervención definitiva de IPaViT-TCC era una intervención cognitiva conductual manualizada de grupos pequeños, diseñada para reducir la VG y mejorar los síntomas depresivos en mujeres con TUS. Se llevó a cabo una adaptación y traducción cultural formal de la WWT para adaptar el contenido de las actividades de la intervención a la población objetivo teniendo en cuenta los siguientes aspectos: 1) Lenguaje: algunos conceptos o palabras que no podían traducirse literalmente fueron reemplazados para que tuvieran más sentido para las participantes; y 2) recursos: se sustituyeron algunos ejercicios, citas e información de recursos para que fueran culturalmente más significativas para la población objetivo.

\section{Condiciones de intervención}

Las 14 participantes se asignaron al azar para recibir la condición experimental o control. Un número aleatorio, generado con el SPSS desde 1-14, fue asignado a las participantes. Los números 1 a 7 fueron asignados a la condición de tratamiento (10 sesiones de intervención IPaViT-TCC en grupo) y números 8 a 14 se asignaron a la condición control. Debido a la naturaleza del estudio, el cegamiento de las participantes no era factible. Los evaluadores no eran cegados a la condición de estudio. No se realizó una evaluación pre-experimental; la investigadora (JT) era responsable de la recogida de datos basales y de seguimiento de las participantes tanto en la condición de intervención como en la de control.

Las mujeres asignadas aleatoriamente a la condición de intervención recibían 10 sesiones de 2 horas durante 5 semanas. La intervención se realizó en centros de tratamiento ambulatorio de adicciones por un psicólogo clínico. La profesional que realizaba la intervención IPaViT-TCC en la condición experimental era una psicóloga clínica (ELL) con más de 30 años de experiencia trabajando con usuarios de drogas, incluyendo la implementación de TCC en grupos. La intervención era manualizada, por lo tanto, las instrucciones figuraban en el manual. Sin embargo, JT se reunió con la psicóloga clínica para discutir la implementación de la terapia manualizada antes de la entrega de cada sesión. Una investigadora (JT) asistió a cada sesión para comprobar la fidelidad de la intervención entregada con relación al manual. Para aumentar la participación, las participantes en el grupo de tratamiento recibieron recordatorios de texto 24 horas antes de cada sesión, un incentivo financiero para asistir a cada sesión y el reembolso de sus gastos de viaje, dado que la intervención integrada se añadía a su tratamiento habitual para el consumo de drogas. Las participantes asignadas al grupo de intervención siguieron recibiendo todos los servicios del tratamiento habitual. Los grupos de control e intervención no recibieron ningún incentivo financiero, recordatorio de texto (sms) ni reembolso de los gastos de viaje para asistir a su tratamiento habitual en el centro comunitario de tratamiento de adicciones.

La condición control recibió el tratamiento habitual proporcionado por el centro de tratamiento ambulatorio de adicciones. El tratamiento habitual consistió en supervisiones quincenales de su uso de sustancias y de su comorbilidad psiquiátrica por profesionales relevantes (psiquiatras, psicólogos y trabajadores sociales) y en el control de la medicación, en el caso de que estuviera recetada. El tratamiento habitual incluyó entrevista motivacional, prevención de recaídas y ayuda psicológica. Aunque el abordaje de la VG no formaba parte del tratamiento habitual, si las participantes en el grupo control reconocieron VG durante una visita de rutina con un profesional, se siguieron las vías de derivación/ tratamiento habitual para asegurar que la participante no estaba en peligro inmediato. En el momento del reclutamiento, las posibles participantes eran informadas de que serían asignadas aleatoriamente a la intervención integrada o el tratamiento habitual, y que si la intervención reducía significativamente la VG, se ofrecería dicha intervención en el futuro a las participantes del grupo control. Esto se explicó a las participantes antes de dar su consentimiento para ser asignadas al azar a los grupos.

Las condiciones de intervención y control fueron implementadas por terapeutas diferentes. Tanto las mujeres de los grupos de intervención como las de control recibieron el tratamiento habitual para el consumo de drogas.

\section{Variables de Resultado}

Todas las participantes completaron una evaluación de línea base administrada por la entrevistadora en una habitación privada de los centros implicados en el estudio. Las entrevistas de seguimiento fueron realizadas en una sala privada o por teléfono si la participante no pudo asistir en persona.

\section{Variables sociodemográficas}

Se recogieron datos sobre la edad, educación, situación laboral, estado civil, estado de salud y características de la pareja.

\section{Violencia de género}

Se utilizaron dos instrumentos para medir la VG - la Composite Abuse Scale (CAS -Escala de Abuso Compuesta; Hegarty, Sheehan y Sconfeld, 1999) y el Psychological Maltreatment of 
Women Inventory (PMWI, Inventario de Maltrato Psicológico de las Mujeres; Tolman, 1999). Además, se empleó la Spouse Specific Assertion/Aggression Scale (SSAAS, Escala de Asertividad/ Agresión Específica al Cónyuge (O’Leary y Curley, 1986) para evaluar la asertividad y la agresión en las relaciones de pareja.

Cualquier VG en el último mes y en los 12 meses se evaluaron mediante el CAS de 30 ítems para identificar el tipo de abuso experimentado: severo combinado (abuso físico y sexual); únicamente abuso físico; abuso físico, abuso emocional y/o acoso; abuso emocional y/o acoso). Se encontró adecuada fiabilidad interna ( $\alpha$ de Cronbach $>.85$ ) para estos 4 factores y las correlaciones ítem-total corregidas eran elevadas (> .5) (Hegarty, Bush y Sheehan, 2005). Cada elemento requiere una respuesta de su frecuencia de ocurrencia en los 12 últimos meses: "nunca", "solamente una vez", "varias veces", “'todos los meses”, "todas las semanas" o "a diario". Se utilizó una puntuación de corte de $\geq 7$ para indicar cualquier tipo de VG.

El PMWI mide el abuso psicológico en el último mes y los 12 meses anteriores (Tolman, 1989). El PMWI contiene 58 ítems que evalúan la frecuencia de abuso emocional/verbal y la dominación/aislamiento desde 1 (nunca) a 5 (muy frecuentemente). La subescala de dominio/aislamiento incluye "elementos relacionados con el seguimiento rígido de los roles sexuales tradicionales, exigencias de sometimiento y aislamiento de recursos", y la subescala de abuso emocional/verbal incluye la "retención de recursos emocionales, comportamiento que podría degradar a las mujeres y el abuso verbal". El PMWI también mostró buena fiabilidad y validez en una muestra de mujeres, principalmente Afro-americanas y blancas ( $\alpha$ de Cronbach $=.88$ ) (Tolman, 1999).

Las relaciones abusivas muestran niveles más bajos de asertividad específica hacia el cónyuge en comparación con las relaciones no abusivas (O’Leary y Curley, 1986). La SSAAS mide el grado de similitud o diferencia en que se identifica la encuestada con cada una de las 29 afirmaciones sobre asertividad y agresión en su relación, con puntuaciones que oscilan entre -3 ( no se parece a mi en absoluto) y +3 (se parece mucho a mî).

La CAS, el PMWI y la SASS no estaban disponibles en español. Por lo tanto, estos instrumentos fueron traducidos del inglés al español por un español nativo familiarizado con el área de investigación, y estas versiones en español fueron nuevamente traducidas (back-translation) al inglés por un hablante nativo de inglés. Estas versiones re-traducidas del inglés fueron enviadas a los autores originales de cada instrumento para su aprobación. La versión en español de la CAS, el PMWI y la SASS fueron aprobados por los autores originales.

\section{Depresión}

La versión en español del Inventario de Depresión de Beck (BDI-II) evaluó los síntomas depresivos en la última semana (Bonicatto, Dew y Soria, 1998). El BDI-II (Beck, Steer y Garbin, 1988) contiene 21 ítems que miden la severidad de la depresión, donde cada respuesta se puntúa de 0 (ausencia de sintomas) a 3 (mayor presencia de sintomas). El BDI-II tiene alta fiabilidad interna ( $\alpha$ de Cronbach $=.88)$ (Bonicatto, Dew y Soria, 1998). Las participan- tes con depresión (puntuación de 19-63, lo que indica depresión moderada a severa) fueron referidas a un psicólogo/psiquiatra en el centro de tratamiento ambulatorio de adicciones.

\section{Calidad de vida y estado de salud}

La calidad de vida y la percepción de la salud se midieron con una escala analógica visual que puntúa desde 0 (menor calidad de vida / salud) a 100 (mejor calidad de vida / salud). La escala ha obtenido buena correlación de fiabilidad test-retest $(r=.87)$ (Boer et al., 2004).

\section{Uso de sustancias}

Por último, la frecuencia y cantidad de uso de alcohol, heroína, cocaína, cannabis y benzodiacepinas durante la última semana se registraron usando una tabla de consumo de sustancias diseñada por el equipo de investigación español basada en la línea de tiempo hacia atrás (TLFB). (Sobell y Sobell, 1992). La fiabilidad test-retest oscila entre $r=+.79 \mathrm{y}+.98$ (Sobell, Maisto, Sobell y Cooper, 1979).

Todos los instrumentos fueron administrados nuevamente por la investigadora 1, 3 y 12 meses después de la intervención. La VG en el pasado mes se evaluó mediante la CAS y el PMWI en todos los momentos del seguimiento. Cualquier VG en los últimos 12 meses fue evaluada utilizando la CAS a los 12 meses de seguimiento. Los síntomas depresivos de la semana pasada se evaluaron con el BDI en todos los momentos del seguimiento.

\section{Fidelidad}

Las participantes del grupo de intervención del estudio completaron un formulario de evaluación al final de cada una de las 10 sesiones de grupo para determinar si el contenido y la información obtenida en cada sesión cumplían los objetivos de cada sesión, con respuestas oscilando desde 1 ( muy en desacuerdo) a 5 (totalmente de acuerdo). Además, se hicieron 3 preguntas al final de cada período de sesiones sobre: 1) lo cómoda que se sentía la participante durante la sesión, oscilando desde 1 ( muy incómoda) a 5 ( muy cómoda); 2) la habilidad del terapeuta, oscilando desde 1 (excelente) a 5 (pobre) y 3) su satisfacción con la sesión, oscilando desde 1 (nada satisfecha) a 5 (muy satisfecha).

\section{Análisis}

Las variables categóricas se calcularon como frecuencias y porcentajes, y las variables continuas como medias y desviaciones típicas. Se realizó análisis de intención de tratar para evitar la pérdida de seguimientos utilizando las puntuaciones de línea base o las últimas puntuaciones del seguimiento. Se emplearon las pruebas exactas de Fisher para comparar las diferencias entre los grupos en los resultados para las variables categóricas, y se usaron pruebas Mann-Whitney para comparar las diferencias entre los grupos en los resultados para las variables continuas. Se calcularon las diferencias pre-post entre línea base y seguimiento. No fue posible controlar los indicadores de resultado de línea base debido al pequeño tamaño muestral. Para este estudio, un valor de $p$ del $5 \%$ o menor se considera estadística- 
mente significativo. La hipótesis era que las participantes que recibieron el WWT tendrían más probabilidades de reducir la VG y los síntomas de la depresión en comparación con las participantes del tratamiento habitual. Se encontró una tasa de abandono diferencial por grupo de tratamiento. Todas las que no completaron el seguimiento habían sido asignadas a la condición control. Se llevó a cabo el análisis de intención de tratar.

\section{Resultados}

\section{Participación y Retención}

La participación en el grupo de intervención era variable (promedio de asistencia a 5 sesiones, rango de 0-9 sesiones).

\section{Datos sociodemográficos}

Las características sociodemográficas de las participantes se recogen en la Tabla 1 . La edad media de las 14 participantes fue 40 años $(S D=8,8)$. En el momento línea base, la mayoría de las participantes eran desempleadas o recibían una pensión de invalidez o un subsidio. Sólo 2 mujeres se habían inyectado drogas en su vida y fueron asignadas al grupo de intervención. En ambos grupos, las mujeres habían ejercido la prostitución en algún momento de su vida. La duración de sus actuales relaciones íntimas era variable; la mayoría de las participantes informó que su actual pareja masculina tenía problemas con el alcohol. Con respecto a las variables de resultado de línea base, los grupos de interven-

Tabla 1

Características sociodemográficas de las participantes ( $n=14$ )

\begin{tabular}{|c|c|c|}
\hline Variables & Intervención (n=7) & Control $(n=7)$ \\
\hline Edad Total & \multicolumn{2}{|c|}{$40(8.81)$} \\
\hline Media (SD) & $42.0(5.56)$ & $39.8(11.61)$ \\
\hline \multicolumn{3}{|l|}{ Conviven } \\
\hline Sola & 1 & 1 \\
\hline Con pareja & 5 & 6 \\
\hline \multicolumn{3}{|l|}{ Empleo } \\
\hline Empleada & 2 & 1 \\
\hline Desempleada & 2 & 2 \\
\hline Pensión invalidez /otros & 0 & 2 \\
\hline Ama de casa & 1 & 2 \\
\hline Heterosexual & 7 & 7 \\
\hline \multicolumn{2}{|l|}{ Se inyecta } & 7 \\
\hline Más de una vez por semana & 2 & 0 \\
\hline \multicolumn{3}{|l|}{ Comercio Sexual } \\
\hline Nunca & 4 & 5 \\
\hline Sí, en el pasado & 2 & 2 \\
\hline Sí, durante el mes pasado & 1 & 0 \\
\hline \multicolumn{3}{|l|}{ Duración de la relación actual } \\
\hline 6- 12 meses & 3 & 2 \\
\hline $13-60$ meses & 1 & 2 \\
\hline+5 años & 3 & 3 \\
\hline \multicolumn{3}{|l|}{ Pareja actual } \\
\hline Problemas con el alcohol & 5 & 5 \\
\hline Problemas con drogas & 1 & 2 \\
\hline \multicolumn{3}{|l|}{ Estado de Salud } \\
\hline $\mathrm{VIH}+$ & 2 & 0 \\
\hline $\mathrm{VHC}+$ & 1 & 2 \\
\hline
\end{tabular}

ción y control eran comparables en casi todas las características a excepción del estado de salud auto-percibida y las respuestas en la SSAAS. En la línea base, las 14 participantes informaron de algún tipo de VG (punto de corte de $\geq 7$ ) pero las asignadas al grupo de intervención describieron ligeramente más maltrato psicológico que las asignadas al grupo control. Las diferencias de línea base en las variables de resultados no pudieron controlarse estadísticamente debido al pequeño tamaño muestral.

\section{Resultados VG}

Escala de Abuso Compuesta (CAS). La Tabla 2 presenta el porcentaje de participantes que informaron de algún incidente de VG, en cada punto de seguimiento y según su condición de tratamiento. Presentamos los datos de aquellas participantes que seguían en una relación de pareja. Todas las mujeres informaron de algún tipo de VG (puntuación CAS igual o mayor que 7) al inicio del estudio. En el grupo de intervención, las participantes informaron de una disminución del $60 \%$ en cualquier VG (física, emocional o acoso) desde la línea base hasta 1 mes ( $n=2$ mujeres informaron de VG) y 3 meses $(n=2$ mujeres informaron de VG) después de la intervención, y una disminución del $80 \%$ a los 12 meses de la intervención, y sólo una mujer informó de VG en el mes anterior. En el grupo control, el porcentaje de participantes que informó de cualquier VG también disminuyó, pero en menor medida, del $100 \%$ en línea base al $71 \%$ al primer mes y al $83 \%$ a los 3 meses después de la intervención, disminuyendo a los 12 meses al $60 \%$ de las participantes que informan haber experimentado cualquier VG.

No había diferencias estadísticamente significativas entre los grupos; no obstante, el grupo de intervención mostró una tendencia de mayores reducciones en la victimización de la VG en todos momentos después de la intervención comparado con el grupo de control. Sin embargo, el tamaño de la muestra puede no haber sido lo suficientemente grande para detectar diferencias.

Inventario de Maltrato Psicológico en las Mujeres (PMWI). Las puntuaciones de maltrato psicológico y asertividad se presentan en la Tabla 2. Una vez más, presentamos sólo los datos para aquellas mujeres que permanecían en una relación de pareja. La subescala de aislamiento/dominancia del PMWI disminuyó desde la línea base $(M=17,20 ; S D=6,14)$ hasta los 12 meses después de la intervención $(M=12,00 ; S D=5,61)$ para las mujeres asignadas al grupo intervención. Para las participantes asignadas al grupo control, la puntuación media de la subescala de aislamiento/dominancia también disminuyó desde la línea base $(M=13,85 ; S D=5,39)$ hasta los 12 meses después de la intervención $(M=8,60 ; S D=2,07)$. Se encontraron diferencias significativas entre los grupos en el seguimiento al mes $(p$ $=, 048)$ y a los 3 meses $(p=, 030)$. La puntuación media en la subescala emocional/verbal del PMWI disminuyó desde la línea base $(M=21,40 ; S D=8,64)$ hasta los 12 meses después de la intervención $(M=11,60 ; S D=1,67)$ para las mujeres asignadas al grupo de intervención. La puntuación media de la subescala emocional/verbal también disminuyó para las participantes del 
Tabla 2

Puntuaciones medias en los instrumentos del estudio (VG, Asertividad) en ambos grupos

\begin{tabular}{|c|c|c|c|c|c|c|c|c|c|}
\hline \multicolumn{3}{|l|}{ Resultado } & $\begin{array}{l}\text { Línea base } \\
\mathrm{N}=14\end{array}$ & $\begin{array}{c}1 \text { mes } \\
\text { seg. } N=12\end{array}$ & $\begin{array}{c}3 \text { meses } \\
\text { seg. } N=11\end{array}$ & $\begin{array}{l}12 \text { meses } \\
\text { seg. } \mathrm{N}=10\end{array}$ & $\begin{array}{c}\text { Valor } p \\
1 \mathrm{~m} \\
\end{array}$ & $\begin{array}{c}\text { Valor } p \\
3 \mathrm{~m}\end{array}$ & $\begin{array}{c}\text { Valor } p \\
12 \mathrm{~m} \\
\end{array}$ \\
\hline \multirow{2}{*}{\multicolumn{2}{|c|}{$\begin{array}{l}\text { Mujeres víctimas de VG } \\
N^{0}(\%) \\
\text { CAS } \geq 7\end{array}$}} & Intervención & $7(100)$ & $2(40)$ & $2(40)$ & $1(20)$ & \multirow{2}{*}{0.558} & \multirow{2}{*}{0.242} & \multirow{2}{*}{0.524} \\
\hline & & Control & $7(100)$ & $5(71)$ & $5(83)$ & $3(60)$ & & & \\
\hline \multirow{4}{*}{$\begin{array}{l}\text { Inventario de } \\
\text { Maltrato } \\
\text { psicológico } \\
\text { a } \\
\text { mujeres } \\
\text { (PMWI) } \\
\text { M (SD) }\end{array}$} & \multirow{2}{*}{$\begin{array}{l}\text { Aislamiento/ } \\
\text { Dominancia }\end{array}$} & Intervención & $\begin{array}{l}17.20 \\
(6.14)\end{array}$ & $\begin{array}{c}8.80 \\
(1.64)\end{array}$ & $\begin{array}{c}8.20 \\
(0.83)\end{array}$ & $\begin{array}{l}12.00 \\
(5.61)\end{array}$ & \multirow{2}{*}{0.048} & \multirow{2}{*}{0.030} & \multirow{2}{*}{0.690} \\
\hline & & Control & $\begin{array}{l}13.85 \\
(5.39)\end{array}$ & $\begin{array}{l}11.71 \\
(3.81)\end{array}$ & $\begin{array}{l}12.33 \\
(3.50)\end{array}$ & $\begin{array}{l}8.60 \\
(2.07)\end{array}$ & & & \\
\hline & \multirow{2}{*}{$\begin{array}{l}\text { Emocional/ } \\
\text { Verbal }\end{array}$} & Intervención & $\begin{array}{l}21.40 \\
(8.64)\end{array}$ & $\begin{array}{l}11.00 \\
(2.00)\end{array}$ & $\begin{array}{l}10.60 \\
(1.34)\end{array}$ & $\begin{array}{l}11.60 \\
(1.67)\end{array}$ & \multirow{2}{*}{0.073} & \multirow{2}{*}{0.126} & \multirow{2}{*}{0.421} \\
\hline & & Control & $\begin{array}{l}18.28 \\
(5.49)\end{array}$ & $\begin{array}{l}14.85 \\
(4.59)\end{array}$ & $\begin{array}{l}17.16 \\
(2.31)\end{array}$ & $\begin{array}{l}12.80 \\
(4.76)\end{array}$ & & & \\
\hline \multirow{4}{*}{$\begin{array}{l}\text { Escala de } \\
\text { Asertividad/ } \\
\text { Agresión Específic } \\
\text { al Cónyuge } \\
\text { (SSAAS) M (SD) }\end{array}$} & \multirow[b]{2}{*}{$\begin{array}{l}\text { Agresividad } \\
\text { ica } \\
\end{array}$} & Intervención & $\begin{array}{l}-8.40 \\
(6.10)\end{array}$ & $\begin{array}{l}-18.80 \\
(7.32)\end{array}$ & $\begin{array}{l}-16.80 \\
(7.19)\end{array}$ & $\begin{array}{l}-12.60 \\
(5.12)\end{array}$ & \multirow[b]{2}{*}{0.030} & \multirow[b]{2}{*}{0.056} & \multirow[b]{2}{*}{0.841} \\
\hline & & Control & $\begin{array}{l}-6.42 \\
(13.62)\end{array}$ & $\begin{array}{c}-1.00 \\
(12.21)\end{array}$ & $\begin{array}{c}1.40 \\
(9.44)\end{array}$ & $\begin{array}{c}-3.60 \\
(14.80)\end{array}$ & & & \\
\hline & \multirow{2}{*}{ Asertividad } & Intervención & $\begin{array}{l}-3.80 \\
(16.39)\end{array}$ & $\begin{array}{l}26.40 \\
(12.44)\end{array}$ & $\begin{array}{c}31.40 \\
(14.62)\end{array}$ & $\begin{array}{l}17.00 \\
(15.41)\end{array}$ & \multirow{2}{*}{0.017} & \multirow{2}{*}{0.056} & \multirow{2}{*}{0.151} \\
\hline & & Control & $\begin{array}{l}7.71 \\
(21.76)\end{array}$ & $\begin{array}{c}15.33 \\
(11.97)\end{array}$ & $\begin{array}{l}19.20 \\
(9.65)\end{array}$ & $\begin{array}{l}16.00 \\
(7.14)\end{array}$ & & & \\
\hline
\end{tabular}

* Nota. Para la PMWI y la SSAAS las medias de línea base son las frecuencias durante el año pasado, otros puntos de tiempo son la frecuencia media durante el mes pasado.

grupo control desde la línea base $(M=18,28 ; S D=5,49)$ hasta los 12 meses después de la intervención $(M=12,80 ; S D=4,76)$. No se encontraron diferencias estadísticamente significativas entre los grupos.

Escala de Asertividad/Agresión Específica al Cónyuge (SSAAS). En el grupo de intervención, las puntuaciones medias de agresividad disminuyeron desde la línea base $(M=-8,40$; $S D=6,10)$ hasta los 12 meses después de la intervención $(M=$ $-12,60 ; S D=5,12$ ). Para las participantes asignados al grupo control, las puntuaciones medias de agresividad aumentaron desde la línea base $(M=-6,42 ; S D=13,62)$ hasta los 12 meses después de la intervención $(M=-3,60 ; S D=14,80)$. Las participantes que recibieron la intervención mostraron mayores disminuciones en la agresividad durante la relación que las que recibían el tratamiento habitual. Se encontró una diferencia estadísticamente significativa entre los grupos un mes $(p=, 030)$ después de la intervención. En el grupo de intervención, la puntuación media de asertividad aumentó desde la línea base $(M=-3,80$; $S D=16,39)$ hasta los 12 meses después de la intervención $(M=$ $17,00 ; S D=15,41)$ en la dirección esperada. Para las participantes asignadas aleatoriamente al grupo control, la puntuación media de asertividad también aumentó desde la línea base $(M$ $=7,71 ; S D=21.76)$ hasta los 12 meses después de la intervención $(M=16,60 ; S D=7,14)$. Todas las participantes mostraron aumentos en la asertividad durante todos los seguimientos. Se encontró una diferencia significativa entre los grupos un mes después de la intervención $(p=, 017)$.

\section{Depresión}

Los resultados de los síntomas depresivos se presentan en la Tabla 3. Las puntuaciones medias del BDI en el grupo de intervención disminuyeron desde la línea base $(M=22,42$; $S D=8,34)$ hasta los 12 meses después de la intervención $(M$ $=14,57 ; S D=8,96)$. En comparación, las puntuaciones medias del BDI en el grupo control disminuyeron desde la línea base $(M=23,42 ; S D=12,73)$ hasta el primer mes después de la intervención $(M=17,00 ; S D=10,36)$, y continuaron disminuyendo hasta los 12 meses $(M=12,28 ; S D=9,60)$. No se encontraron diferencias significativas entre los grupos.

\section{Estado de salud y Calidad de vida}

El estado de salud y la calidad de vida autopercibidos se presentan en la Tabla 3. En el grupo de intervención, la puntuación media de estado de salud autopercibido disminuyó desde la línea base $(M=61,42 ; S D=22,45)$ hasta el primer mes después de la intervención $(M=48,71 ; S D=33,78)$, pero aumentó a los $3(M=76,42 ; S D=15,73)$ y 12 meses después de la intervención $(M=62,85 ; S D=23,42)$ en comparación con la línea base. En el grupo control, las puntuaciones medias se mantuvieron estables en todos los puntos del seguimiento (línea base $M=49,01 ; S D=21,07$ ) hasta 12 meses después de la intervención $(M=48,57 ; S D=25,44)$. Las mujeres asignadas al grupo de intervención informaron de un mejor estado de salud autopercibido que las mujeres del grupo control, pero no hubo diferencias significativas entre los grupos. 
Tabla 3

Puntuaciones medias en los instrumentos del estudio (Depresión, Estado de Salud y Calidad de vida) en ambos grupos

\begin{tabular}{|c|c|c|c|c|c|c|c|c|}
\hline Resultado & & Línea base & $\begin{array}{c}1 \mathrm{mes} \\
\text { seg. }\end{array}$ & $\begin{array}{c}3 \text { meses } \\
\text { seg. }\end{array}$ & $\begin{array}{c}12 \text { meses } \\
\text { seg. }\end{array}$ & $\begin{array}{c}\text { Valor } p \\
1 \mathrm{~m} \\
\end{array}$ & $\begin{array}{c}\text { Valor } p \\
3 \mathrm{~m}\end{array}$ & $\begin{array}{c}\text { Valor } p \\
12 \mathrm{~m} \\
\end{array}$ \\
\hline \multirow{2}{*}{$\begin{array}{l}\text { Depresión } \\
\text { BDI } \\
\text { M (SD) }\end{array}$} & Intervención & $\begin{array}{l}22.42 \\
(8.34)\end{array}$ & $\begin{array}{l}14.42 \\
(8.16)\end{array}$ & $\begin{array}{l}11.28 \\
(5.18)\end{array}$ & $\begin{array}{l}14.57 \\
(8.96)\end{array}$ & \multirow{2}{*}{0.535} & \multirow{2}{*}{0.535} & \multirow{2}{*}{0.620} \\
\hline & Control & $\begin{array}{l}23.42 \\
(12.73)\end{array}$ & $\begin{array}{l}17.00 \\
(10.36)\end{array}$ & $\begin{array}{c}15.28 \\
(10.95)\end{array}$ & $\begin{array}{l}12.28 \\
(9.60)\end{array}$ & & & \\
\hline \multirow{2}{*}{$\begin{array}{l}\text { Estado de } \\
\text { salud } \\
M \text { (SD) }\end{array}$} & Intervención & $\begin{array}{l}61.42 \\
(22.45)\end{array}$ & $\begin{array}{c}48.71 \\
(33.78)\end{array}$ & $\begin{array}{c}76.42 \\
(15.73)\end{array}$ & $\begin{array}{l}62.85 \\
(23.42)\end{array}$ & \multirow{2}{*}{0.209} & \multirow{2}{*}{0.165} & \multirow{2}{*}{0.805} \\
\hline & Control & $\begin{array}{l}49.01 \\
(21.07)\end{array}$ & $\begin{array}{c}55.71 \\
(13.04)\end{array}$ & $\begin{array}{c}55.00 \\
(23.80)\end{array}$ & $\begin{array}{c}48.57 \\
(25.44)\end{array}$ & & & \\
\hline \multirow{2}{*}{$\begin{array}{l}\text { Calidad } \\
\text { de Vida } \\
\text { M (SD) }\end{array}$} & Intervención & $\begin{array}{l}40.71 \\
(23.52)\end{array}$ & $\begin{array}{c}29.78 \\
(27.27)\end{array}$ & $\begin{array}{c}55.71 \\
(17.18)\end{array}$ & $\begin{array}{c}59.28 \\
(20.08)\end{array}$ & \multirow{2}{*}{0.209} & \multirow{2}{*}{1} & \multirow{2}{*}{0.620} \\
\hline & Control & $\begin{array}{l}42.42 \\
(18.06)\end{array}$ & $\begin{array}{c}49.00 \\
(12.79)\end{array}$ & $\begin{array}{c}52.85 \\
(19.11)\end{array}$ & $\begin{array}{c}54.28 \\
(19.02)\end{array}$ & & & \\
\hline
\end{tabular}

La calidad de vida de las participantes del grupo de intervención mejoró en todos los seguimientos, con excepción del seguimiento del primer mes, desde la línea base $(M=$ $40,71 ; S D=23,52)$, hasta 3 meses después de la intervención $(M=55,71 ; S D=17,18)$ y hasta 12 meses después de la intervención $(M=59,28 ; S D=20,08)$. La puntuación media de las participantes del grupo control también aumentó desde la línea base $(M=42,42 ; S D=18,06)$ hasta los 12 meses después de la intervención $(M=54,28 ; S D=19,02)$ pero en menor grado que el grupo de intervención. No se encontraron diferencias estadísticas entre los grupos para este resultado.

\section{Uso de sustancias}

El promedio de número de días de consumo de alcohol disminuyó desde la línea base $(M=22,28 ; S D=40,35)$ hasta el seguimiento de 1 mes después de la intervención $(M=6,42$; $S D=12,83$ ) para las participantes del grupo de intervención. El promedio de número de días de consumo de alcohol aumentó para las participantes del grupo control desde la línea de base $(M=6,28 ; S D=13,79)$ hasta el primer mes de seguimiento después de la intervención $(M=10,0 ; S D=17,29)$. Se encontró una diferencia significativa entre los grupos en el uso autoinformado de alcohol $(p=, 035)$. No se encontraron diferencias significativas entre los grupos en el número de días de consumo de alcohol en los otros momentos de seguimiento. Para otras sustancias, no se encontraron diferencias significativas.

\section{Evaluación de las sesiones}

Las participantes completaron una evaluación de calidad de la sesión al final de cada una de las sesiones. Las participantes informaron que los conocimientos adquiridos durante las sesiones eran altos, con una puntuación media de 4,5 ( $S D=0,50)$-la puntuación máxima era 5 . Con respecto a sentirse cómoda du- rante las sesiones, el $83 \%$ de las participantes informaron que se sentían "muy cómodas" durante las sesiones, el $90 \%$ consideró que la actuación del terapeuta era "excelente", y el 80\% evaluó las sesiones en general como "excelentes".

\section{Discusión}

Aunque las mujeres con TUS informan de altos índices de VG y de síntomas de depresión, pocos estudios han examinado la eficacia de las intervenciones de TCC para reducir la victimización por VG en esta población. Algunos de estos estudios tenían seguimientos cortos (Gilbert et al., 2006) que no permiten evaluar si los beneficios se mantienen a largo plazo. Hasta donde nosotras sabemos, este ensayo piloto y de viabilidad es el primer estudio que valora los resultados para las víctimas femeninas de VG que asisten a tratamientos por abuso de sustancias, 12 meses después de la intervención. Este ensayo piloto muestra resultados esperanzadores en términos de la viabilidad y efectividad inicial de la intervención. A los 12 meses de la intervención, las participantes que recibieron la intervención y permanecían con sus parejas mostraron reducciones en la frecuencia de la violencia psicológica recibida y mejoras en sus relaciones, con mayor asertividad y menos agresividad en su comunicación con la pareja. También se ha detectado una tendencia de mayores reducciones en la VG física. Las participantes del grupo control también mostraron reducciones en la VG (física y psicológica). Esto podría ser debido a que también recibían tratamiento para el consumo de drogas, que ha podido producir mejoras en sus relaciones de pareja y por lo tanto, mejora en los resultados, aunque las mejorías fueron menores en este grupo que en el grupo de intervención. No obstante, se encontraron diferencias significativas entre los grupos para el maltrato psicológico. La intervención IPaViT-TCC fue más eficaz que el tratamiento habitual para reducir el maltrato 
psicológico, como el aislamiento/dominancia, al mes y a los 3 meses después de la intervención pero esto no se mantuvo a largo plazo (12 meses de seguimiento). También se encontraron diferencias significativas entre los grupos en asertividad y agresividad en la relación al mes después de la intervención. Estos resultados sugieren que las mujeres que recibían la intervención IPaViT-TCC eran más propensas a informar de menos abuso psicológico y a mejorar sus relaciones que las del tratamiento habitual en todos los seguimientos. Esto puede ser debido a que la intervención TCC ofrece a las mujeres las habilidades necesarias para re-evaluar sus relaciones y esto a su vez cambió la dinámica del maltrato psicológico. Nuestros resultados son consistentes con otros estudios en términos de la eficacia en reducir VG (Gilbert et al., 2006; Cohen, Field, Campbell y Hien, 2013). Un ensayo reciente de TCC comparado con el tratamiento habitual en atención primaria informó de reducciones en los síntomas depresivos en mujeres víctimas de VG (Hegarty et al., 2013). La intervención IPaViT-TCC no favoreció la reducción de síntomas de depresión comparado con el tratamiento habitual en adicciones. Las participantes de ambos grupos informaron de una reducción de síntomas depresivos y una mejora en la calidad de vida. Por lo tanto, una única sesión que aborde el estado de ánimo negativo podría ser insuficiente para tratar la compleja comorbilidad depresiva entre las usuarias de drogas. Ensayos futuros podrían tener que mejorar la intervención para abordar los síntomas de depresión. La intervención IPaViT-TCC demostró una alta fidelidad y buena asistencia así como altas tasas de participación y retención, lo que sugiere que este tipo de intervención es factible de implementar en centros comunitarios de tratamiento de adicciones para abordar la victimización por VG. Por otra parte, resultados del estudio sugieren muy buena aceptación entre las participantes, quienes indicaron un alto nivel de satisfacción con la intervención y con su terapeuta. Las participantes también pensaban que habían adquirido nuevos conocimientos.

Alrededor de 15 de las mujeres evaluadas eran elegibles para participar, en el sentido de que habían sido víctimas de VG en el último mes y mantenían una relación de pareja. Estudios futuros podrían considerar extender este periodo de tiempo para facilitar el reclutamiento. La principal limitación de este estudio es el pequeño tamaño de muestra, por lo que la interpretación de los resultados debe realizarse con precaución. Los resultados del presente estudio pueden utilizarse para informar de los parámetros de un futuro ensayo definitivo de la intervención. Una limitación es que los datos de resultados son autoinformados. Otra limitación era la variable participación de las mujeres en las sesiones de grupo, a pesar de los incentivos financieros, recordatorios de texto (sms) y el reembolso de gastos de viaje. La administración de contingencias basada en los principios del condicionamiento operante, como el uso de incentivos (Higgins et al., 1994), fue especialmente efectivo para mejorar la supervisión del tratamiento y sus resultados en esta población (Higgins, Alessi y Dantona, 2002). Como la literatura ha mostrado, estas estrategias pueden utilizarse y parecen ser eficaces. A pesar de esto, estas variables deben ser controladas en futuros ensayos piloto, ya que en la literatura sobre el tema se ha mostrado que afectan los resultados de forma consistente. Desafortunadamente, las diferencias de línea base se identificaron después de la aleatorización. Las diferencias de línea base en las variables de resultados no pudieron controlarse estadísticamente debido al pequeño tamaño muestral. Las diferencias de línea base en el consumo de alcohol pueden afectar la evolución. Sin embargo, todas las participantes recibieron tratamiento para el trastorno por uso de alcohol, y el problema del alcohol y su evolución se supervisaron desde el centro comunitario de tratamiento en ambos grupos.

Es necesario realizar un futuro ensayo más robusto para replicar estos resultados y establecer conclusiones firmes acerca de la eficacia de la intervención en comparación con el tratamiento habitual. La comparación coste-efectividad de la intervención debe considerarse con respecto al tratamiento habitual en futuros ensayos.

\section{Conclusiones}

Los resultados de la viabilidad de la prueba piloto sugieren cierto apoyo inicial para la intervención TCC de 10 sesiones de grupo para las víctimas de VG que recibieron tratamiento por consumo de drogas. Esta intervención de grupo, IPaViT-CBT, diseñada para reducir la VG y mejorar los síntomas de depresión, proporciona una alternativa cuando nos enfrentamos a la toma de decisiones sobre cómo abordar la victimización por VG entre mujeres con TUS. Se requiere un ensayo más robusto para replicar estos resultados.

\section{Agradecimientos}

Este trabajo fue financiado por las subvenciones: RD12/0028/009 del Fondo de Investigaciones Sanitarias, Instituto Carlos III-FEDER y un acuerdo de cooperación entre el "Departament de Salut" y el "Parc de Salut Mar de Barcelona" para desarrollar e implementar una intervención de grupo para reducir la violencia de género en las usuarias de drogas. Todas las autoras contribuyeron igualmente a este trabajo. Todas las autoras revisaron el manuscrito críticamente y participaron en la interpretación de los datos.

\section{Conflictos de interés}

Todas las autoras declaran que no tienen ningún conflicto de interés.

\section{Referencias}

Beck, A. T., Steer, R. A. y Garbin, M.G. (1988). Psychometric properties of the Beck Depression Inventory: Twenty-five years of evaluation. Clinical Psychology Review, 8, 77-100. 
Boer, A. G., Lanschot, J. J., Stalmeier, P. F., Sandick, J. W., Hulscher, J. B., Haes, J. C. y Sprangers, M. A. (2004). Is a single-item visual analogue scale as valid, reliable and responsive as multi-item scales in measuring quality of life? Quality of Life Research, 13, 311-320.

Bonicatto, S., Dew, A. M. y Soria, J. J. (1998). Analysis of the psychometric properties of the Spanish version of the Beck Depression Inventory in Argentina. Psychiatry Research, 79, 277-285.

Bourgois, P., Prince, B. y Moss, A. (2004). The everyday violence of hepatitis $\mathrm{C}$ among young women who inject drugs in San Francisco. Human Organization, 63, 253264.

Campbell, J. C. (2002). Health consequences of intimate partner violence. Lancet, 359, 1331-1336. doi: 10.1016/ s0140-6736(02) 08336-8

Campbell, J. C., Baty, M. L., Ghandour, R. M., Stockman, J. K., Francisco, L. y Wagman, J. (2008). The intersection of intimate partner violence against women and HIV/AIDS: A review. International Journal of Injury Control and Safety Promotion, 15, 221-231. doi: 10.1080/17457300802423224

Carpenter, K. M., Aharonovich, E., Smith, J. L., Iguchi, M. Y. y Nunes, E. V. (2006). Behavior therapy for depression in drug dependence (BTDD): Results of a stage Ia therapy development pilot. The American Journal of Drug and Alcohol Abuse, 32, 541-548. doi: 10.1080/00952990600919450

Castillo-Camiglia, A., Pizarro, E., Luengo, D. y Soto-Brandt, G. (2014). Consumo de alcohol y autoinforme de eventos violentos en Chile. Adicciones, 26, 46-53.

Cohen, L. R., Field, C., Campbell, A. N. y Hien, D. A. (2013). Intimate partner violence outcomes in women with PTSD and substance use: A secondary analysis of NIDA Clinical Trials Network "Women and Trauma" Multi-site Study. Addictive Behaviors, 38, 2325-2332. doi: 10.1016/j.addbeh.2013.03.006

Cohen, L. R. y Hien, D. A. (2006). Treatment outcomes for women with substance abuse and PTSD who have experienced complex trauma. Psychiatric Services, 57, 100-106. doi: 10.1176/appi.ps.57.1.100

Connelly, C. D., Hazen, A. L., Baker-Ericzen, M. J., Landsverk, J. y Horwitz, S. M. (2013). Is screening for depression in the perinatal period enough? The co-occurrence of depression, substance abuse, and intimate partner violence in culturally diverse pregnant women. Journal of Women's Health (Larchmt), 22, 844-852. doi: 10.1089/ jwh.2012.4121

El-Bassel, N., Gilbert, L., Witte, S., Wu, E. y Chang, M. (2011). Intimate partner violence and HIV among drug-involved women: contexts linking these two epidemics-challenges and implications for prevention and treatment. Substance Use Eं Misuse, 46, 295-306. doi: $10.3109 / 10826084.2011 .523296$
El-Bassel, N., Gilbert, L., Wu, E., Go, H. y Hill, J. (2005). Relationship between drug abuse and intimate partner violence: a longitudinal study among women receiving methadone. American Journal of Public Health, 95, 465470. doi: 10.2105/ajph.2003.023200

Fearday, F., Clark, C. y Edington, M. (Eds.) (2001). Triad Women's Project Group Facilitator Manual and Workbook. Tampa, FL: Louis de la Porte Florida Mental Health Institute, University of South Florida.

Fowler, D. N. y Faulkner, M. (2011). Interventions targeting substance abuse among women survivors of intimate partner abuse: a meta-analysis. Journal of Substance Abuse Treatment, 41, 386-98. doi: 10.1016/j.jsat.2011.06.001.

Gilbert, L., El-Bassel, N., Manuel, J., Wu, E., Go, H., Golder, S., .. Sanders, G. (2006). An integrated relapse prevention and relationship safety intervention for women on methadone: testing short-term effects on intimate partner violence and substance use. Violence and Victims, 21, 657-672.

Gilchrist, G., Blazquez, A. y Torrens, M. (2011). Psychiatric, behavioural and social risk factors for HIV infection among female drug users. AIDS and Behavior, 15, 18341843. doi: 10.1007/s10461-011-9991-1

Gilchrist, G., Blazquez, A. y Torrens, M. (2012). Exploring the relationship between intimate partner violence, childhood abuse and psychiatric disorders among female drug users. Advances in Dual Diagnosis, 5, 46-58.

Harris, M. y Community Connections Trauma Work Group (Eds.) (1998). Trauma Recovery and Empowerment: A Clinician's guide for working with women in groups. New York: Community Connections, Inc.

Hearn, K. D., O’Sullivan, L. F., El-Bassel, N. y Gilbert, L. (2005). Intimate partner violence and monogamy among women in methadone treatment. AIDS and Behavior, 9, 177-186. doi: 10.1007/s10461-005-3899-6

Hegarty, K., Bush, R. y Sheehan, M. (2005). The composite abuse scale: further development and assessment of reliability and validity of a multidimensional partner abuse measure in clinical settings. Violence and Victims, 20, 529547.

Hegarty, K., O’Doherty, L., Taft, A., Chondros, P., Brown, S., Valpied, J., . . Gunn, J. (2013). Screening and counselling in the primary care setting for women who have experienced intimate partner violence (WEAVE): a cluster randomised controlled trial. Lancet, 382, 249-258. doi: 10.1016/s0140-6736(13)60052-5

Hegarty, K., Sheehan, M. y Schonfeld, C. (1999). A Multidimensional Definition of Partner Abuse: Development and Preliminary Validation of the Composite Abuse Scale. Journal of Family Violence, 14, 399-415.

Higgins, S. T., Alessi, S. M. y Dantona, R. L. (2002). Voucher-based incentives. A substance abuse treatment innovation. Addictive Behaviors, 27, 887-910. 
Higgins, S. T., Budney, A. J., Bickel, W. K., Foerg, F. E., Donham, R. y Badger, G. J. (1994). Incentives improve outcome in outpatient behavioral treatment of cocaine dependence. Archives of General Psychiatry, 51, 568-576.

Howard, L. M., Trevillion, K., Khalifeh, H., Woodall, A., Agnew-Davies, R. y Feder, G. (2010). Domestic violence and severe psychiatric disorders: prevalence and interventions. Psychological Medicine, 40, 881-893. doi: $10.1017 / \mathrm{s} 0033291709991589$

Illangasekare, S. L., Burke, J. G., McDonnell, K. A. y Gielen, A. C. (2013). The impact of intimate partner violence, substance use, and HIV on depressive symptoms among abused low-income urban women. Journal of Interpersonal Violence, 28, 2831-2848. doi: 10.1177/0886260513488682

Kaysen, D., Dillworth, T. M., Simpson, T., Waldrop, A., Larimer, M. E. y Resick, P. A. (2007). Domestic violence and alcohol use: trauma-related symptoms and motives for drinking. Addictive Behaviors, 32, 1272-1283. doi: 10.1016/j.addbeh.2006.09.007

Liebschutz, J. M., Mulvey, K. P. y Samet, J. H. (1997). Victimization among substance-abusing women. Worse health outcomes. Archives of Internal Medicine, 157, 1093-1097.

McKinney, C. M., Caetano, R., Rodriguez, L. A. y Okoro, N. (2010). Does alcohol involvement increase the severity of intimate partner violence? Alcoholism, Clinical and Experimental Research, 34, 655-658. doi: 10.1111/j.15300277.2009.01134.x

Miller, D. y Guidry, L. (Eds.) (2001). Addictions and Trauma Recovery: Healing the Body, Mind, and Spirit. New York: W.W. Norton and Co.

Miller, B. A., Downs, W. R. y Testa, M. (1993). Interrelationships between victimization experiences and women's alcohol use. Journal of Studies on Alcohol. Supplement, 11, 109-117.

Najavits, L. M. (Ed.) (2009). Psychotherapies for trauma and substance abuse in women: review and policy implications. Trauma, Violence $\mathcal{E}$ Abuse, 10, 290-8. doi: $10.1177 / 1524838009334455$.

Najavits, L. M. (2002). Seeking Safety: A Treatment Manual for PTSD and Substance Abuse. Nueva York: Guildford Press.

Najavits, L. M., Sonn, J., Walsh, M. y Weiss, R. D. (2004). Domestic violence in women with PTSD and substance abuse. Addictive Behaviors, 29, 707-715. doi: 10.1016/j. addbeh.2004.01.003

O’Leary, K. D. y Curley, A.D. (1986). Assertion and family violence: Correlates of Spouse Abuse. Journal of Marital and Family Therapy, 12, 281- 289.

Okuda, M., Olfson, M., Hasin, D., Grant, B. F., Lin, K. H. y Blanco, C. (2011). Mental health of victims of intimate partner violence: results from a national epidemiologic survey. Psychiatric Services, 62, 959-962. doi: 10.1176/ appi.ps.62.8.959

Peters, E. N., Khondkaryan, E. y Sullivan, T. P. (2012). Associations between expectancies of alcohol and drug use, severity of partner violence, and posttraumatic stress among women. Journal of Interpersonal Violence, 27, 21082127. doi: 10.1177/0886260511432151

Singer, M. (1996). A dose of drugs, a touch of violence, a case of AIDS: Conceptualizing the SAVA syndemic. Free Inquiry in Creative Sociology, 24, 99-110.

Sobell, L. S. y Sobell, M. B. (1992). Timeline follow-back: A technique for assessing self-reported ethanol consumption. Measuring Alcohol Consumption: Psychological and Biological Methods, 41-72.

Sobell, L. C., Maisto, S. A., Sobell, M. B. y Cooper, A. M. (1979). Reliability of alcohol abusers' self-reports of drinking behavior. Behaviour Research and Therapy, 17, 157-160.

Testa, M., Livingston, J. A. y Hoffman, J. H. (2007). Does sexual victimization predict subsequent alcohol consumption? A prospective study among a community sample of women. Addictive Behaviors, 32, 2926-2939. doi: 10.1016/j.addbeh.2007.05.017

Tirado-Muñoz, J., Gilchrist, G., Farré, M., Hegarty, K. y Torrens, M. (2014). The efficacy of cognitive behavioural therapy and advocacy interventions for women who have experienced intimate partner violence: A systematic review and meta-analysis. Annals of Medicine, 11, 1-20. doi:10.3109/07853890.2014.941918

Tolman, R.M. (1989). The development of a measure of psychological maltreatment of women by their male partners. Violence and Victims, 4, 159-177.

Tolman, R. M. (1999). The validation of the Psychological Maltreatment of Women Inventory. Violence and Victims, 14, 25-37.

Torrens, M., Gilchrist, G. y Domingo-Salvany, A. (2011). Psychiatric comorbidity in illicit drug users: substance-induced versus independent disorders. Drug and Alcohol Dependence, 113, 147-156. doi: 10.1016/j.drugalcdep.2010.07.013

Trevillion, K., Oram, S., Feder, G. y Howard, L. M. (2012). Experiences of domestic violence and mental disorders: a systematic review and meta-analysis. PLoS One, 7, e51740. doi: 10.1371/journal.pone.0051740

Wagner, K. D., Hudson, S. M., Latka, M. H., Strathdee, S. A., Thiede, H., Mackesy-Amiti, M. E. y Garfein, R. S. (2009). The effect of intimate partner violence on receptive syringe sharing among young female injection drug users: an analysis of mediation effects. AIDS and Behavior, 13, 217-224. doi: 10.1007/s10461-007-9309-5

World Health Organization. (2013). Responding to intimate partnerviolence and sexualviolence against women: WHOclinical and policy guidelines. Recuperado de http:/ / apps.who. int/iris/bitstream/10665/85240/1/9789241548595_ eng.pdf?ua $=1$ 